\title{
EFFECT OF ORGANIC MANURES AND CHEMICAL FERTILIZERS ON FOENICULUM VULGARE, MILL AND CARUM CARVI, L.
}

Souzan M. Ibrahim ${ }^{1}$, Hosny M. El-Labban ${ }^{2}$, Fayez I. Mohamed ${ }^{3}$ and Neveen M. Naga ${ }^{2}$

${ }^{1}$ Department of Pharmacognosy, Faculty of Pharmacy, Tanta University, Tanta, Egypt

${ }^{2}$ Department of Horticulture, Faculty of Agriculture, Tanta University, Tanta, Egypt

${ }^{3}$ Department of Horticulture Res. Inst., Agricultural Research Center, Egypt

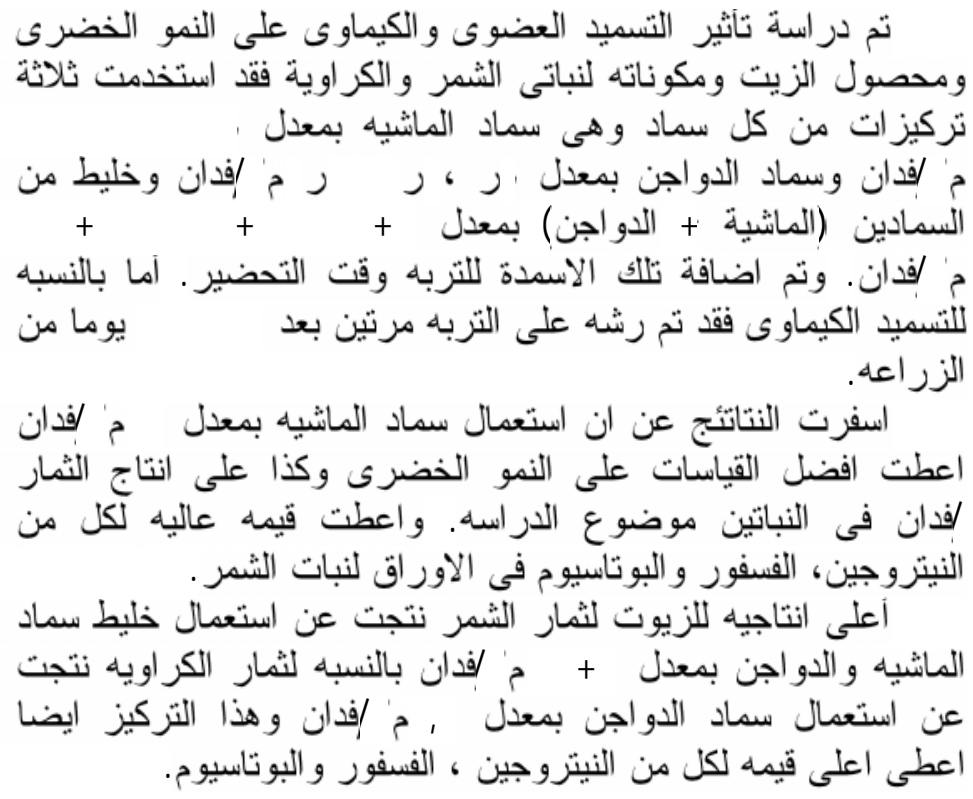

The effect of organic manures and chemical fertilizers on the vegetative growth and oil production and composition for both plants were studied. The experiments were carried out during two seasons of 2001/2002 and 2002/2003.

Three concentration from each of farmyard manure, FYM $(8,12$ and $\left.16 \mathrm{~m}^{3} / \mathrm{fed}\right)$, chicken manure, $C M\left(0.8,1.2\right.$ and $\left.1.6 \mathrm{~m}^{3} / \mathrm{fed}\right)$ and

Received in 12/3/2006 \& Accepted in 4/6/2006 
mixed manures of FYM $+C M\left(4+0.4,6+0.6\right.$ and $\left.8+0.8 \mathrm{~m}^{3} / \mathrm{fed}\right)$ were added at the time of soil preparation. The chemical fertilizers $N$ and $K$ were used as soil dressing two times after 50 and 75 days from planting.

The results indicated that the use of FYM, $16 \mathrm{~m}^{3} / \mathrm{fed}$ treatment produced high vegetative growth and high fruit yield/fed in both plants as well as high values of NPK for fennel plant.

The high oil yield was produced from the use of the mixed treatment FYM +CM $4+0.4 \mathrm{~m}^{3} /$ fed and by the use of $C M, 1.2$ $\mathrm{m}^{3} / \mathrm{fed}$ in case of fennel and caraway plants respectively. The dose of CM, $1.2 \mathrm{~m}^{3} /$ fed with caraway also produce high values of NPK.

\section{INTRODUCTION}

Foeniculum vulgare, Mill (Fennel) and Carum carvi, L. (Caraway), Apiaceae family, are important medicinal and aromatic plants. ${ }^{1}$ They are used as popular flavoring agents in culinary preparations, bread, pastry and confectionery. ${ }^{2}$

Fennel volatile oil is commonly used as antimicrobial, galactagogue, for estrogenic activities and as a remedy for jaundice and menstrual troubles. Fennel plant occupies a high position in exportation list of medicinal plants. ${ }^{3-6}$

Caraway fruits are traditionally used as a remedy for dyspepsia, intestinal colic and antispasmodic. ${ }^{7 \& 8}$

It was reported that the use of organic fertilizers as chicken, poultry, farmyard or cattle manures increased the vegetative growth as well as the contents of several aromatic plants. ${ }^{9-16}$

This investigation was conducted us to study the effect of application of organic manures and chemical Fertilizers on the vegetative growth of Foeniculum vulgare, Mill and Carum carvi, L. plants as well as on oil yield and compostion of their fruits.

\section{MATERIAL AND METHODS}

This study was carried out during the period 2001-2003 in two successive seasons at the Farm of Medicinal and Aromatic Plants, Gemmiza (A.R.C.) Gharbieah Governorate.

The fruits of Foeniculim vulgare, Mill and Carum Carvi, L were sown on November $15^{\text {th }}$ in the first season and November $1^{\text {st }}$ in the second one.

The organic manures used were three doses of each of farmyard manure (FYM) 8, 12 and $16 \mathrm{~m}^{3} / \mathrm{fed}$., chichen manure $(\mathrm{CM})$ 0.8, 1.2 and 1.6 $\mathrm{m}^{3} /$ fed. and mixed manures (FYM + CM) $4+0.4,6+0.6,8+0.8 \mathrm{~m}^{3} /$ fed .

Before planting the calculated amount of organic manures and calcium superphosphate were added at the time of soil preparation. Chemical fertilizers ( $\mathrm{N}$ in the form of ammonium sulphate $130 \mathrm{~kg} / \mathrm{fed}$ and $\mathrm{k}$ as potassium sulphate $100 \mathrm{~kg} / \mathrm{fed}$ ) 
were added also with calcium superphosphate as soil dressing after 50 and 75 days from planting. The experiments were designed in a complete randomize blocks with three replicates.

Control treatment was used without any addition. The plant height $(\mathrm{cm})$, number of branches and umbles/ plant, plant fresh weight $(\mathrm{g})$, umbles dry weight (g), weight of 100 fruits $(\mathrm{g})$, weight of fruits yield/plant (g) and /fed. (Ton) were recorded at the full blooming of both plants.

The percentage of $\mathrm{N}, \mathrm{P}$ and $\mathrm{K}$ elements were determined according to the literature procedures. ${ }^{17-19}$ Volatile oils content of the fruits were determined by water distillation method $^{20}$ and GLC analysed using Hewlett Packard 5890 with flame ionization detection that was fitted with capillary column, coated with carbowax $20 \mathrm{M} \times 0.2 \mathrm{~min}$. The operating conditions were injector temperature $250^{\circ}, \quad$ detector temperature $300^{\circ}$. Nitrogen was used as a carrier gas with flow rate $1 \mathrm{ml} / \mathrm{min}$, for hydrogen was 30 $\mathrm{ml} / \mathrm{min}$. and for air was $300 \mathrm{ml} / \mathrm{min}$. The peaks were recorded and the areas under peaks were determined using HP-integrator. Series II apparatus. The oil components were identified by comparing their retention time to that of the authentic compounds.

The mean values of the treatments were compared by L.S.D. test according to Snedecor and
Cochran.

\section{RESULTS AND DISCUSSION}

The data in Tables (1 and 2) showed that all fertilization treatments significantly increased all the vegetative parameters for fennel plant in comparing with the control group for both seasons.

The highest values were noticed in both seasons with $16 \mathrm{~m}^{3} / \mathrm{fed} \mathrm{FYM}$ treatments for all parameters except with number of umbles/plant and the weight of 100 fruits. The two latter parameters showed indicative values with the mixed manures FYM + CM at $4: 0.4$ and $8: 0.8 \mathrm{~m}^{3} / \mathrm{fed}$ respectively in both seasons. Our results were in accordance with the findings of Mohamed and Ahmed, ${ }^{9}$ Migahed and El-Kased ${ }^{22}$ and Abd-ElSalam. $^{23}$

The recorded data in Table (3) indicated that the volatile oils content in fennel fruits were increased as a result of fertilization during the two experimental seasons. The most pronounced effect resulted from the mixed manures $\mathrm{FYM}+\mathrm{CM}$ at the concentration ratio of $4+0.4 \mathrm{~m}^{2} / \mathrm{fed}$. The increase in volatile oil content was probably due to the increment in the metabolic activities. These results were in agreement with those of Jacoub $^{13}$ on thyme and $\mathrm{Aly}^{24}$ on fennel. 
Table 1: Effect of fertilizaion treatments on the vegetative growth of Foeniculum vulgare, Mill in the first season 2001/2002.

\begin{tabular}{|c|c|c|c|c|c|c|c|c|}
\hline \multirow[b]{2}{*}{$\begin{array}{l}\text { Treatments } \\
\mathrm{m}^{3} / \mathrm{fed}\end{array}$} & \multicolumn{8}{|c|}{ Effect on } \\
\hline & $\begin{array}{l}\text { Plant height } \\
\text { "cm" }\end{array}$ & $\begin{array}{c}\text { No. of } \\
\text { branches/ } \\
\text { plant }\end{array}$ & $\begin{array}{c}\text { Plant } \\
\text { fresh } \\
\text { weight }(\mathrm{g})\end{array}$ & $\begin{array}{c}\text { No. of } \\
\text { umbels/ } \\
\text { plant }\end{array}$ & $\begin{array}{l}\text { Weight of } \\
\text { umbels/ } \\
\text { plant (g) }\end{array}$ & $\begin{array}{l}\text { Weight of } \\
100 \text { fruits }(\mathrm{g})\end{array}$ & $\begin{array}{c}\text { Fruits } \\
\text { weight/ } \\
\text { plant (g) }\end{array}$ & $\begin{array}{c}\text { Fruit } \\
\text { yield/fed. } \\
\text { (ton) }\end{array}$ \\
\hline Control & 116.67 & 9.50 & 516.67 & 38.8 & 87.3 & 0.86 & 37.58 & 0.563 \\
\hline FYM, 8 & 127.00 & 14.33 & 520.67 & 52.3 & 132.0 & 0.89 & 59.50 & 0.892 \\
\hline 12 & 135.00 & 14.17 & 591.33 & 39.0 & 122.3 & 0.93 & 53.00 & 0.783 \\
\hline 16 & 186.67 & 14.77 & 881.67 & 39.2 & 162.0 & 1.03 & 117.53 & 1.763 \\
\hline CM, 0.8 & 184.33 & 12.83 & 662.17 & 48.2 & 112.7 & 0.97 & 81.27 & 1.219 \\
\hline 1.2 & 144.67 & 14.50 & 689.0 & 52.5 & 142.0 & 1.01 & 103.00 & 1.545 \\
\hline 1.6 & 157.67 & 13.33 & 813.33 & 36.2 & 152.0 & 0.89 & 110.27 & 1.654 \\
\hline $\mathrm{FYM}+\mathrm{CM}, 4+0.4$ & 158.33 & 14.3 & 729.17 & 56.2 & 135.3 & 0.90 & 98.18 & 1.473 \\
\hline $6+0.6$ & 163.33 & 11.17 & 745.0 & 45.8 & 132.7 & 0.92 & 68.92 & 1.034 \\
\hline $8+0.8$ & 135.17 & 12.83 & 735.83 & 47.2 & 137.7 & 1.07 & 99.88 & 1.498 \\
\hline NPK & 170.33 & 11.17 & 795.83 & 42.3 & 114.0 & 1.00 & 82.71 & 1.241 \\
\hline L.S.D. (5\%) & 6.51 & 1.99 & 15.43 & 4.89 & 36.42 & 0.139 & 10.32 & 0.069 \\
\hline L.S.D. (1\%) & 8.85 & 2.67 & 17.93 & 6.05 & 41.73 & 0.189 & 14.71 & 0.094 \\
\hline
\end{tabular}


Table 2: Effect of Fertilizaion treatments on the vegetative growth of Foeniculum vulgare, Mill in the second season 2002/2003.

\begin{tabular}{||c|c|c|c|c|c|c|c|c||}
\hline \multirow{2}{*}{$\begin{array}{c}\text { Treatments } \\
\left(\mathrm{m}^{3} / \mathrm{fed}\right)\end{array}$} & $\begin{array}{c}\text { Plant } \\
\text { height } \\
\text { "cm" }\end{array}$ & $\begin{array}{c}\text { No. of } \\
\text { branches/ } \\
\text { plant }\end{array}$ & $\begin{array}{c}\text { Plant } \\
\text { fresh } \\
\text { weight }(\mathrm{g})\end{array}$ & $\begin{array}{c}\text { No. of } \\
\text { umbels/ } \\
\text { plant }\end{array}$ & $\begin{array}{c}\text { Weight of } \\
\text { mbels/ } \\
\text { plant (g) }\end{array}$ & $\begin{array}{c}\text { Weight } \\
\text { of 100 } \\
\text { fruits (g) }\end{array}$ & $\begin{array}{c}\text { Fruits } \\
\text { weight/ } \\
\text { plant (g) }\end{array}$ & $\begin{array}{c}\text { Fruit } \\
\text { yield/fed. } \\
\text { (ton) }\end{array}$ \\
\hline Control & 161.33 & 8.50 & 478.43 & 34.7 & 97.8 & 1.12 & 42.67 & 0.640 \\
FYM, 8 & 190.17 & 12.67 & 695.42 & 50.7 & 174.7 & 1.14 & 113.68 & 1.705 \\
12 & 167.17 & 11.50 & 675.75 & 50.0 & 142.3 & 1.22 & 105.12 & 1.577 \\
16 & 195.89 & 11.87 & 1087.10 & 81.5 & 198.7 & 1.29 & 146.41 & 2.196 \\
CM, 0.8 & 194.50 & 11.03 & 896.67 & 71.5 & 188.5 & 0.98 & 123.33 & 1.850 \\
1.2 & 190.50 & 11.83 & 808.33 & 77.5 & 161.3 & 1.14 & 126.97 & 1.905 \\
1.6 & 188.50 & 11.25 & 963.75 & 67.2 & 193.5 & 1.20 & 142.71 & 2.141 \\
FYM + CM, + & 190.00 & 11.50 & 956.67 & 87.0 & 182.9 & 1.18 & 134.58 & 2.019 \\
& 181.50 & 10.17 & 773.33 & 66.7 & 190.0 & 1.25 & 140.49 & 2.108 \\
NPK & 169.17 & 11.33 & 733.33 & 67.7 & 182.2 & 1.34 & 134.57 & 2.019 \\
\hline L.S.D. (5) & 187.83 & 12.00 & 949.50 & 86.0 & 191.0 & 1.27 & 141.23 & 2.119 \\
L.S.D. (1\%) & 4.40 & 1.75 & 56.96 & 4.66 & 44.88 & 0.34 & 5.39 & 0.225 \\
\hline
\end{tabular}


Table 3: Effect of fertilization treatments on volatile oil percentage, oil yield/plant and oil yield/feddan of Foeniculum vulgare, Mill fruits during the two seasons of 2001/2002 and 2002/2003.

\begin{tabular}{||c|c|c|c|c|c|c|}
\hline \multirow{2}{*}{$\begin{array}{c}\text { Treatments } \\
\left(\mathrm{m}^{3} / \mathrm{fed}\right)\end{array}$} & \multicolumn{3}{|c|}{ First seasons 2001/2002 } & \multicolumn{3}{c|}{ Second seasons 2002/2003 } \\
\cline { 2 - 7 } & Oil \% & $\begin{array}{c}\text { Oil yield/ } \\
\text { Plant (ml) }\end{array}$ & $\begin{array}{c}\text { Oil yield/ } \\
\text { fed. (L) }\end{array}$ & Oil \% & $\begin{array}{c}\text { Oil yield/ } \\
\text { plant (ml) }\end{array}$ & $\begin{array}{c}\text { Oil yield/ } \\
\text { fed. (L) }\end{array}$ \\
\hline Control & 1.00 & 0.38 & 5.65 & 1.10 & 0.47 & 7.05 \\
FYM, 8 & 1.22 & 0.73 & 10.90 & 1.38 & 1.57 & 23.55 \\
12 & 2.80 & 0.44 & 6.60 & 2.69 & 2.20 & 32.95 \\
16 & 1.66 & 1.95 & 29.25 & 1.54 & 2.25 & 33.80 \\
CM, 0.8 & 1.40 & 1.13 & 17.00 & 1.50 & 1.85 & 27.75 \\
1.2 & 1.68 & 1.73 & 25.95 & 1.76 & 2.24 & 33.55 \\
1.6 & 2.00 & 2.21 & 33.10 & 1.92 & 2.74 & 41.10 \\
FYM + CM, 4+0.4 & 2.88 & 2.63 & 39.45 & 2.80 & 3.66 & 56.55 \\
& 1.96 & 1.35 & 20.25 & 1.62 & 2.28 & 34.15 \\
NPK & 1.69 & 1.69 & 25.30 & 1.86 & 2.51 & 37.60 \\
NPK & 2.68 & 2.22 & 33.25 & 2.44 & 3.45 & 51.70 \\
\hline L.S.D. (5\%) & 0.346 & 0.396 & 2.21 & 0.219 & 099 & 2.11 \\
L.S.D. (1\%) & 0.491 & 0.544 & 3.40 & 0.372 & 1.33 & 2.26 \\
\hline
\end{tabular}

GLC analysis of the volatile oil fruits samples during the second seasons Table (4) indicated that $\alpha-$ pinene, limonene, linalool, methyl chavicol and anethol were identified in all treatments. These results were in agreement with the results obtaned by Guenther ${ }^{2}$ and $\mathrm{Sakr}^{14}$ on mentha and Mohsen ${ }^{26}$ on sweet basil.

Methyl chavicol was the main component in all the tested treatments as it showed the highest values in relation to the other components. The highest percentage of methyl chavicol was obtained from CM $1.6 \mathrm{~m}^{3} / \mathrm{fed}$ treatment NPK was the most effective treatments on the linalool and anethol content for all the tested oils. The highest values of $\alpha$-pinene were observed with the mixed treatment of $6+0.6 \mathrm{~m}^{3} / \mathrm{fed} \mathrm{FYM}+\mathrm{CM}$, while that of limonene were noticed at FYM, 8 $\mathrm{m}^{3} /$ fed.

The effect of fertilization treatments on nitrogen, phosphorous and potassium percentage of fennel leaves in the two experimental seasons are presented in Table (5). The highest level of both $\mathrm{N}$ and $\mathrm{P}$ were observed with FYM at 16 $\mathrm{m}^{3} /$ fed treatment, while CM, 0.8 $\mathrm{m}^{3} /$ fed treatment showed the highest $\mathrm{K}$ percentage.

As regard the effect of fertilization treatments on the vegetative growth of caraway during both seasons, it is 
Table 4: Effect of different fertilization treatments on the percentage of volatile oil components of Foeniculum vulgare, Mill fruits during the second season of 2002/2003.

\begin{tabular}{|c|c|c|c|c|c|c|c|c|c|c|c|c|c|}
\hline \multirow{3}{*}{ No } & \multirow{3}{*}{$\begin{array}{l}\text { Retent- } \\
\text { ion } \\
\text { time }\end{array}$} & \multirow{3}{*}{ Identification } & \multicolumn{11}{|c|}{$\%$ of components in the treatments $\left(\mathrm{m}^{3} / \mathrm{fed}\right)$} \\
\hline & & & \multirow{2}{*}{ Control } & \multicolumn{3}{|c|}{ FYM } & \multicolumn{3}{|c|}{$\mathrm{CM}$} & \multicolumn{3}{|c|}{$\mathrm{FYM}+\mathrm{CM}$} & \multirow{2}{*}{ NPK } \\
\hline & & & & 8 & 12 & 16 & 0.8 & 1.2 & 1.6 & $4+0.4$ & $6+0.6$ & $8+0.8$ & \\
\hline 1 & 3.295 & $\alpha$-pinene & 0.679 & 0.999 & 0.683 & 0.454 & 0.454 & 0.813 & 0,476 & 0.580 & 1.279 & 0.377 & 0.483 \\
\hline 2 & 3.709 & Unknown & 0.111 & ---- & 0.1036 & --- & --- & --- & 0.168 & 0.256 & --- & 0.252 & --- \\
\hline 3 & 4.200 & Fenchone & --- & 1.202 & 0.785 & --- & --- & ---- & --- & 0.194 & --- & --- & 1.53 \\
\hline 4 & 4.573 & Unknown & 0.491 & ---- & 0.218 & --- & --- & --- & --- & --- & --- & --- & --- \\
\hline 5 & 5.529 & Unknown & 0.354 & ---- & --- & --- & --- & --- & --- & 0.141 & --- & --- & --- \\
\hline 6 & 5.972 & Limonene & 10.722 & 12.794 & 8.714 & 7.818 & 6.841 & 6.068 & 7.711 & 7.030 & 7.803 & 5.752 & 4.074 \\
\hline 7 & 6.257 & Unknown & 0.469 & 0.495 & 0.455 & --- & --- & --- & --- & --- & 0.178 & 0.412 & 1.216 \\
\hline 8 & 8.967 & Linalool & 2.991 & 1.521 & 2.711 & 4.772 & 4.739 & 3.343 & 3.208 & 3.614 & 3.048 & 4.374 & 4.822 \\
\hline 9 & 15.754 & Methyl chavicol & 79.353 & 80.285 & 82.216 & 82.866 & 83.274 & 83.077 & 85.057 & 84.913 & 83.065 & 84.144 & 81.927 \\
\hline 10 & 16.671 & Unknown & 1.870 & --- & 0.195 & 0.710 & 0.366 & 1.067 & 0.781 & 0.709 & 1.641 & 0.372 & 0.166 \\
\hline 11 & 19.351 & Anethol & 2.346 & 1.658 & 0.587 & 0.891 & 0.813 & 2.613 & 0.574 & 1.502 & 1.988 & 0.420 & 2.691 \\
\hline 12 & 27.801 & Unknown & 0.246 & 0.186 & 0.656 & 1.152 & 0.932 & 0.185 & ---- & 0.232 & --- & 0.905 & 0.017 \\
\hline 13 & 29.004 & Unknown & 0.176 & 0.369 & --- & 0.206 & 0.644 & 0.492 & 1.241 & 0.720 & 0.410 & 0.106 & 0.028 \\
\hline
\end{tabular}


Table 5: Effect of fertilization treatments on N, P and $\mathrm{K}$ percentage of Foeniculum vulgare, Mill leaves during the two seasons of 2001/2002 and 2002/2003.

\begin{tabular}{||c|c|c|c|c|c|c|}
\hline \multirow{2}{*}{$\begin{array}{c}\text { Treatments } \\
\left(\mathrm{m}^{3} / \mathrm{fed}\right)\end{array}$} & \multicolumn{3}{|c|}{ First seasons 2001/2002 } & \multicolumn{3}{c|}{ Second seasons $2002 / 2003$} \\
\cline { 2 - 7 } & $\mathrm{N} \%$ & $\mathrm{P} \%$ & $\mathrm{~K} \%$ & $\mathrm{~N} \%$ & $\mathrm{P} \%$ & $\mathrm{~K} \%$ \\
\hline Control & 1.89 & 0.16 & 1.4 & 1.81 & 0.12 & 0.9 \\
FYM, 8 & 1.83 & 0.19 & 1.3 & 2.04 & 0.16 & 1.4 \\
12 & 3.70 & 0.35 & 3.3 & 3.09 & 0.35 & 2.4 \\
16 & 3.64 & 0.28 & 1.9 & 3.03 & 0.27 & 2.0 \\
$\mathrm{CM}, 0.8$ & 3.42 & 0.22 & 3.4 & 2.46 & 0.2 & 2.5 \\
1.2 & 2.81 & 0.18 & 1.44 & 2.39 & 0.19 & 2.0 \\
1.6 & 2.57 & 0.22 & 1.2 & 2.85 & 0.2 & 1.7 \\
FYM + CM, 4+0.4 & 3.46 & 0.29 & 2.3 & 2.68 & 0.29 & 2.3 \\
& 2.43 & 0.19 & 1.2 & 2.03 & 0.2 & 1.2 \\
NPK & 2.12 & 0.2 & 1.4 & 2.7 & 0.25 & 1.9 \\
& 2.28 & 0.28 & 1.9 & 2.36 & 0.22 & 1.8 \\
\hline
\end{tabular}

tabulated in Tables (6 and 7). The results indicated that all the treatments were increased the studied vegetative parameters. Farmyard manure was the most effective treatment when applied at the rate of $16 \mathrm{~m}^{3} / \mathrm{fed}$. Our results were in accordance with the findings of $\mathrm{Fiad}^{26}$ on caraway, Aly et al. ${ }^{27}$ and Abd ElKader $^{28}$ on coriander and anise.

Table (8) clearly showed that the volatile oils percentage and yields were significantly increased with the application of different fertilization treatments. In all cases supplying plants with $\mathrm{CM}$ at $1.2 \mathrm{~m}^{3} / \mathrm{fed}$ gave significantly higher oil percentage and yield in both seasons. The previous results were agreed with those obtained by Abd El-Salam, ${ }^{23}$ Hammam $^{29}$ and Abd El-Wahab ${ }^{30}$ on fennel, anise and nigella.
Carvone, limonene and $\alpha$-pinene were noticed in all treatments of the GLC volatile oil analysis of the samples taken from the second season Table (9). The table also clearly indicated that carvone was the main component of caraway oil and the highest percent of it was obtained from the mixed manures FYM $+\mathrm{CM}$, $4+0.4 \mathrm{~m}^{3} /$ fed while the lowest percentage was obtained from FYM, $12 \mathrm{~m}^{3} /$ fed treatments. Limonene was the second component of the oil, it was observed in high percentage by the NPK treatment. This treatment also showed higher percentage of $\alpha$ pinene which exhibited relatively small percentage in the oil.

Our results were in agreement with those of Khattab and Omer. ${ }^{31}$

The effect of fertilization treatments on the NPK in the leaves ofcaraway is presented in Table (10). 
Table 6: Effect of fertilizaion treatments on the vegetative growth of Carum carvi, L. in the first season 2001/2002.

\begin{tabular}{||c|c|c|c|c|c|c|c|c||}
\hline \multirow{2}{*}{$\begin{array}{c}\text { Treatments } \\
\left(\mathrm{m}^{3} / \mathrm{fed}\right)\end{array}$} & $\begin{array}{c}\text { Plant } \\
\text { height } \\
\text { "cm" }\end{array}$ & $\begin{array}{c}\text { No. of } \\
\text { branches/ } \\
\text { plant }\end{array}$ & $\begin{array}{c}\text { Plant } \\
\text { fresh } \\
\text { weight }(\mathrm{g})\end{array}$ & $\begin{array}{c}\text { No. of } \\
\text { umbels/ } \\
\text { plant }\end{array}$ & $\begin{array}{c}\text { Weight of } \\
\text { mbels/ } \\
\text { plant (g) }\end{array}$ & $\begin{array}{c}\text { Weight } \\
\text { of 100 } \\
\text { fruits (g) }\end{array}$ & $\begin{array}{c}\text { Fruits } \\
\text { weight/ } \\
\text { plant (g) }\end{array}$ & $\begin{array}{c}\text { Fruit } \\
\text { yield/fed. } \\
\text { (ton) }\end{array}$ \\
\hline Control & 101.67 & 9.00 & 319.0 & 47.33 & 70.67 & 0.576 & 48.67 & 0.584 \\
FYM, 8 & 112.17 & 10.50 & 355.33 & 80.83 & 90.67 & 0.561 & 63.50 & 0.762 \\
12 & 110.00 & 9.50 & 550.0 & 66.67 & 104.00 & 0.578 & 73.67 & 0.884 \\
16 & 121.67 & 11.67 & 658.33 & 131.00 & 134.33 & 1.073 & 99.33 & 1.192 \\
CM, 0.8 & 116.67 & 11.00 & 401.67 & 82.88 & 80.50 & 0.572 & 58.00 & 0.695 \\
1.2 & 112.67 & 10.00 & 371.67 & 56.67 & 126.17 & 0.614 & 92.00 & 1.104 \\
1.6 & 111.00 & 11.00 & 341.0 & 72.67 & 89.17 & 0.627 & 67.67 & 0.812 \\
FYM + CM, 4+0.4 & 112.67 & 8.50 & 450.00 & 78.83 & 92.67 & 0.749 & 69.33 & 0.832 \\
& 106.67 & 10.00 & 323.33 & 66.50 & 71.00 & 0.634 & 55.33 & 0.664 \\
NPK & 115.00 & 9.17 & 463.33 & 81.33 & 76.67 & 0.645 & 54.67 & 0.656 \\
\hline L.S.D. (5\%) & 116.00 & 9.67 & 296.67 & 75.17 & 80.00 & 0.699 & 55.00 & 0.660 \\
L.S.D. (1\%) & 8.09 & 1.17 & 30.98 & 9.38 & 12.92 & 0.073 & 9.11 & 0.086 \\
\hline \hline
\end{tabular}


Table 7: Effect of fertilizaion treatments on the vegetative growth of Carum carvi, L. in the second season 2002/2003.

\begin{tabular}{|c|c|c|c|c|c|c|c|c|}
\hline \multirow[b]{2}{*}{$\begin{array}{l}\text { Treatments } \\
\left(\mathrm{m}^{3} / \mathrm{fed}\right)\end{array}$} & \multicolumn{8}{|c|}{ Effect on } \\
\hline & $\begin{array}{l}\text { Plant } \\
\text { height } \\
\text { "cm" }\end{array}$ & $\begin{array}{c}\text { No. of } \\
\text { branches/ } \\
\text { plant }\end{array}$ & $\begin{array}{l}\text { Plant fresh } \\
\text { weight }(\mathrm{g})\end{array}$ & $\begin{array}{c}\text { No. of } \\
\text { umbels/ } \\
\text { plant }\end{array}$ & $\begin{array}{c}\text { Weight } \\
\text { of mbels/ } \\
\text { plant }(\mathrm{g})\end{array}$ & $\begin{array}{l}\text { Weight } \\
\text { of } 100 \\
\text { fruits }(\mathrm{g})\end{array}$ & $\begin{array}{c}\text { Fruits } \\
\text { weight/ } \\
\text { plant (g) }\end{array}$ & $\begin{array}{c}\text { Fruit } \\
\text { yield/fed. } \\
\text { (ton) }\end{array}$ \\
\hline Control & 117.33 & 10.83 & 325.0 & 52.17 & 74.00 & 0.632 & 54.88 & 0.658 \\
\hline FYM, 8 & 124.83 & 10.50 & 365.33 & 85.17 & 95.70 & 0.805 & 69.13 & 0.829 \\
\hline 12 & 122.90 & 10.57 & 564.67 & 68.33 & 108.60 & 1.219 & 79.1 & 0.949 \\
\hline 16 & 134.83 & 12.50 & 676.0 & 136.72 & 139.33 & 1.274 & 105.7 & 1.268 \\
\hline CM, 0.8 & 122.67 & 11.17 & 406.83 & 85.17 & 86.50 & 0.843 & 62.17 & 0.758 \\
\hline 1.2 & 125.67 & 10.33 & 386.00 & 61.67 & 132.07 & 1.094 & 97.23 & 1.166 \\
\hline 1.6 & 129.83 & 10.83 & 347.00 & 77.00 & 91.58 & 0.981 & 82.23 & 0.986 \\
\hline $\mathrm{FYM}+\mathrm{CM}, 4+0.4$ & 125.00 & 11.73 & 442.20 & 79.83 & 92.92 & 0.815 & 72.43 & 0.869 \\
\hline $6+0.6$ & 129.50 & 10.67 & 332.82 & 74.17 & 102.23 & 0.779 & 54.67 & 0.656 \\
\hline $8+0.8$ & 125.00 & 10.50 & 483.83 & 84.00 & 82.95 & 0.967 & 58.4 & 0.701 \\
\hline NPK & 122.83 & 10.33 & 356.5 & 79.89 & 87.33 & 0.788 & 60.63 & 0.727 \\
\hline L.S.D. (5\%) & 8.07 & 1.55 & 26.340 & 11.14 & 14.84 & 0.236 & 8.65 & 0.271 \\
\hline L.S.D. (1\%) & 10.97 & 2.11 & 35.646 & 15.14 & 20.17 & 0.287 & 12.47 & 0.373 \\
\hline
\end{tabular}


Table 8: Effect of fertilization treatments on volatile oil percentage, oil yield/plant and oil yield/feddan of Carum carvi, L. fruits during the two seasons of 2001/2002 and 2002/2003.

\begin{tabular}{||c|c|c|c|c|c|c||}
\hline \multirow{2}{*}{$\begin{array}{c}\text { Treatments } \\
\left(\mathrm{m}^{3} / \mathrm{fed}\right)\end{array}$} & \multicolumn{2}{|c|}{ First seasons 2001/2002 } & \multicolumn{2}{c|}{ Second seasons 2002/2003 } \\
\cline { 2 - 7 } & $\begin{array}{c}\text { Oil } \\
\%\end{array}$ & $\begin{array}{c}\text { Oil yield/ } \\
\text { plant (ml) }\end{array}$ & $\begin{array}{c}\text { Oil } \\
\text { yield/ } \\
\text { fed. (L) }\end{array}$ & $\begin{array}{c}\text { Oil } \\
\%\end{array}$ & $\begin{array}{c}\text { Oil yield/ } \\
\text { plant } \\
(\mathrm{ml})\end{array}$ & $\begin{array}{c}\text { Oil } \\
\text { yield/ } \\
\text { fed. (L) }\end{array}$ \\
\hline Control & 1.10 & 0.54 & 6.44 & 1.20 & 0.66 & 7.92 \\
FYM, 8 & 2.24 & 1.42 & 17.04 & 2.08 & 1.44 & 17.24 \\
12 & 2.10 & 1.55 & 18.56 & 1.92 & 1.52 & 18.24 \\
16 & 2.48 & 2.46 & 29.56 & 2.12 & 2.24 & 26.92 \\
CM, 0.8 & 1.96 & 1.14 & 13.68 & 2.00 & 1.26 & 15.16 \\
1.2 & 3.00 & 2.54 & 30.48 & 2.80 & 2.43 & 29.16 \\
1.6 & 2.88 & 1.95 & 23.4 & 2.66 & 2.19 & 26.32 \\
FYM + CM, 4+0.4 & 1.50 & 1.04 & 15.52 & 1.78 & 1.29 & 15.48 \\
$6+0.6$ & 2.50 & 1.38 & 16.64 & 2.22 & 1.21 & 14.56 \\
$8+0.8$ & 2.20 & 1.20 & 14.44 & 1.94 & 1.13 & 13.6 \\
NPK & 2.76 & 1.65 & 19.8 & 2.75 & 1.61 & 19.28 \\
\hline L.S.D. (5\%) & 0.37 & 0.163 & 2.77 & 0.31 & 0.199 & 3.21 \\
L.S.D. (1\%) & 0.48 & 0.223 & 2.96 & 0.42 & 0.277 & 3.54 \\
\hline
\end{tabular}


Table 9: Effect of different fertilization treatments on the volatile oil components of Carum carvi, $L$. fruits during the second season of 2002/2003.

\begin{tabular}{|c|c|c|c|c|c|c|c|c|c|c|c|c|c|}
\hline \multirow{3}{*}{ No } & \multirow{3}{*}{$\begin{array}{c}\text { Retent- } \\
\text { ion } \\
\text { time }\end{array}$} & \multirow{3}{*}{$\begin{array}{l}\text { Identificat- } \\
\text { ion }\end{array}$} & \multicolumn{11}{|c|}{$\%$ of the identified components in treatments $\mathrm{m}^{3} / \mathrm{fed}$} \\
\hline & & & \multirow{2}{*}{ Control } & \multicolumn{3}{|c|}{ FYM } & \multicolumn{3}{|c|}{$\mathrm{CM}$} & \multicolumn{3}{|c|}{$\mathrm{FYM}+\mathrm{CM}$} & \multirow{2}{*}{ NPK } \\
\hline & & & & 8 & 12 & 16 & 0.8 & 1.2 & 1.6 & $4+0.4$ & $6+0.6$ & $8+0.8$ & \\
\hline 1 & 3.895 & $\alpha$-pinene & 0.268 & 0.227 & 0.276 & 0.269 & 0.282 & 1.188 & 0.236 & 0.244 & 0.222 & 0.237 & 0.374 \\
\hline 2 & 5.455 & Limonene & 19.274 & 19.427 & 23.783 & 18.274 & 18.437 & 16.824 & 20.141 & 15.843 & 17.330 & 20.807 & 26.187 \\
\hline 3 & 6.303 & Unknown & 0.685 & 0.733 & 0.333 & 1.012 & 0.905 & --- & --- & 0.445 & --- & 0.460 & --- \\
\hline 4 & 9.841 & Unknown & 0.397 & 0.615 & 0.614 & 0.619 & 0.343 & 0.495 & 0.451 & 0.496 & 0.335 & 0.366 & --- \\
\hline 5 & 11.970 & Unknown & --- & --- & 0.059 & --- & 0.087 & --- & --- & 0.075 & 0.054 & 0.060 & 0.060 \\
\hline 6 & 14.463 & Unknown & 0.537 & 0.653 & 0.520 & 0.545 & 0.540 & 0.412 & 0.485 & 0.473 & 0.420 & 0.528 & 0.471 \\
\hline 7 & 18.463 & Carvone & 77.811 & 77.945 & 71.652 & 78.462 & 79.083 & 81.745 & 77.992 & 82.220 & 81.353 & 79.945 & 72.035 \\
\hline 8 & 23.292 & Unknown & --- & --- & 0.053 & 0.037 & ---- & --- & --- & --- & --- & --- & --- \\
\hline 9 & 24.673 & Unknown & 0.140 & 0.189 & ---- & 0.130 & 0.107 & 0.065 & 0.139 & --- & 0.064 & 0.109 & 0.125 \\
\hline 10 & 28.732 & Unknown & 0.097 & 0.138 & 0.748 & --- & --- & 0.098 & 0.051 & --- & 0.088 & --- & ---- \\
\hline
\end{tabular}


Table 10: Effect of fertilization treatment on $\mathrm{n}, \mathrm{p}$ and $\mathrm{k}$ percentage of Carum carvi, L. leaves during the two seasons of 2001/2002 and 2002/2003.

\begin{tabular}{||c|c|c|c|c|c|c||}
\hline \multirow{2}{*}{$\begin{array}{c}\text { Treatments } \\
\mathrm{m}^{3} / \mathrm{fed}\end{array}$} & \multicolumn{3}{|c|}{ First seasons 2001/2002 } & \multicolumn{3}{c|}{ Second seasons 2002/2003 } \\
\cline { 2 - 7 } & $\mathrm{N} \%$ & $\mathrm{P} \%$ & $\mathrm{~K} \%$ & $\mathrm{~N} \%$ & $\mathrm{P} \%$ & $\mathrm{~K} \%$ \\
\hline Control & 2.09 & 0.19 & 2.0 & 2.27 & 0.25 & 2.4 \\
FYM, 8 & 2.20 & 0.30 & 402 & 2.73 & 0.32 & 4.5 \\
12 & 2.30 & 0.24 & 3.9 & 2.20 & 0.22 & 3.6 \\
16 & 3.63 & 0.32 & 4.8 & 2.73 & 0.27 & 4.4 \\
$\mathrm{CM}, 0.8$ & 3.39 & 0.33 & 4.7 & 3.20 & 0.31 & 4.5 \\
1.2 & 3.84 & 0.37 & 4.9 & 3.98 & 0.33 & 4.6 \\
1.6 & 3.10 & 0.25 & 3.1 & 3.76 & 0.30 & 3.8 \\
FYM + CM, 4+0.4 & 2.80 & 0.30 & 3.7 & 3.43 & 0.32 & 3.9 \\
& 3.46 & 0.23 & 3.1 & 3.18 & 0.28 & 3.6 \\
$\mathrm{NPK}$ & 3.30 & 0.25 & 3.2 & 3.93 & 0.28 & 3.8 \\
\hline
\end{tabular}

$\mathrm{CM}, 1.2 \mathrm{~m}^{3} / \mathrm{fed}$ treatment was the most effective one, as it showedhigher percentage of NPK content.

\section{REFERENCES}

1- G. E. Trease and W. C. Evans, "Text Book of Pharmacognosy", $13^{\text {th }}$ Ed. Bailiere Tindall, London NWI 70x, England, 1989, pp. 435-440

2- E. Guenther, "The Essential Oils", Vols. I and IV, $4^{\text {th }}$ Ed., D. van Nostrand Company, Inc. Princeton, New Jersey, Toronto, New York, London (1961).

3- I. Morelli, E. Bonar, A. M. Pagni and P. E. Tomei, Schoool of Specialization Science and Technology of Medicinal Plants, Fac. of Pharmacy, University of
Pisa, Food and Agriculture Organization of the United Nations Rome, 1983, pp. 16-63.

4- F. T. K. Hussein, Medicinal Plants in Libya, 462 (1985).

5- Y. Albert, "Encyclopidia of Common Natural Ingredients Used in Food, Drugs, and Cosmetics", Awiley-Interscience Publication, New York, 1980, pp. 169-171.

6- M. Y. M. Ali, "Physiological Studies on Foeniculum vulgare Mill. Plant Under Sinai Conditions", M.Sc. Thesis, Fac. Agric., Cairo Univ., Egypt (2002).

7- J. Lawless, "The Encylopoaedia of Essential Oils, the Complete Guide to the Use of Aromatics in Aromatherapy Herbalism", Health \& Well Being ElementShaftesbury, Dorse-Rochport, 
Massachusetts Brisbane, Queensland (1992).

8- B. Golambosi and P. Peura, J. Essent. Oil Res., 8, 389 (1996).

9- A. A. Mohamed and M. E. Ahmed, Minia J. of Agric. Res. \& Dev., 22, 221 (2002).

10- M. A. Abdou and M. A. H. Mahmoud, J. Agric. Sci. Mansoura Univ., 28, 3857 (2003).

11- U. R. Khandkar and K. B. Nigam, Ind. of Agric., Sci., 66, 549 (1996), (Hort. Abst., 67, 3440).

12- E. A. E. El-Ghadban, "Effect of Some Organic and Inorganic Fertilizer on Growth, Oil Yield and Chemical Composition of Spearmint and Majoram Plants", Ph.D. Thesis, Fac. Agric., Cairo Univ., Egypt (1998).

13- R. W. Jacoub, "Effect Of Some Organic and in Organic Fertilizers on Growth, Oil Yield and Chemical Composition of Ocimum basilicum L. and Thymus vulgaris L. Plants", Ph.D. Thesis, Fac. Agric., Cairo Univ., Egypt (1999).

14- W. R. Sakr, " Effect of Some Organic and Inorganic Fertilizers on Mentha pipertia.", M.Sc. Thesis, Fac., Agric., Cairo Univ., Egypt (2001).

15- S. A. El-Gendy, A. M. Hosni, S. S. Ahmed and M. Salri, Ann. Agric. Sci., Ain Shams Univ., Cairo, 46, 319 (2001).

16- M. Helmy and S. S. Zarad, J. Agric. Sci. Mansoura Univ., 28, 3911 (2003).
17- C. A. Black, "Methods of Soil Analysis", Part 1 and 2. Soil Sci. Soc. Amer. Inc. Pulp., Madison, Wise U.S.A (1983).

18- F. S. Watanabe and S. R. Olsen, Test of an ascorbic acid method for determining phosphours in water and $\mathrm{NaHCO}_{3}$ extracts from soil. Soil Science Society Proceeding, 1965, pp. 677-678.

19- L. A. Richards, "Diagnosis and Improvement of Saline and Alkaline Oils", U.S.D.A. Agric. Hand Book No. 60. Gov. Print. Off (1954).

20- S. I. Balbaa, S. H. Hilal and A. Y. Zaki, "Medicinal Plants Constituents", $3^{\text {rd }}$ Ed., General Organization for Univ. Books, Cairo, Egypt, 1981, p. 644.

21- G. W. Snedecor and W. G. Cochran, "Statistical Methods", $7^{\text {th }}$ Iowa State, Univ. Press. Amer. Iowa. U.S.A (1980).

22- H. A. M. Migahed and F. A. ElKased, J. Appl. Sci., 13, 327 (1998).

23- I. Z. Abd El-Salam, "Physiological Studies on Fennel Plant (Foeniculum vulgare Mill)", Ph.D. Thesis, Fac. Agric., Cairo Univ., Egypt (1999).

24- M. S. Aly, J. Agric. Sci., Mansoura Univ., 28, 3215 (2003).

25- M. M. Mohsen, "Sweet Basil Herb and Oil Production as Affected by Chemical and Organic Fertilization", M.Sc. Thesis, Fac. Agric., Cairo Univ., Egypt (2002). 
26- A. M. Fiad, "Physiological Study on Caraway", M.Sc. Thesis, Fac. Agric., Zagazig Univ. (1993).

27- S. A. Aly, R. K. S. Tomar and K. N. Maurya, Bhartiya Krishi Anusandhan Patika, 9, 241 (1994), (Hort. Abst., 66, 4434).

28- A. Abd-El-Kader, "Nitrogen Nutrition of Fennel (Foeniculum vulgare, Mill) and Anise (Pimpinella anisum, L.) and Their Effects on Growth and Essential Oil Contents", M.Sc. Thesis, Fac. Agric., Assiut Univ., Egypt (1999).
29- K. A. Hmmam, "Effect of Nitrogenous Fertilization and Irrigation on Growth, Yield and Active Constituents of Anise Plants (Pimpinella anisum, L.)", M.Sc. Thesis, Fac. Agric., Cairo Univ., Egypt (1996).

30- M. A. Abd El-Wahab, "Effect of Chemical Fertilization on Cuminum cyminum L. and Nigella sativa L. Plants Under North Sinai Condition", M.Sc. Thesis, Fac. Agric., Cairo Univ., Egypt (1997).

31- M. E. Khattab and E. A. Omer, J. Hort., 26, 249 (1999). 